\title{
Arterial catecholamine and enzyme histochemistry in migraine
}

\author{
C. W. M. ADAMS, C. C. ORTON, AND K. J. ZILKHA \\ From the Department of Pathology, Guy's Hospital Medical School, Department of Neurology, \\ King's College Hospital, and The National Hospital, Queen Square, London
}

The purpose of this communication is to present details of a histochemical investigation of superficial temporal arteries biopsied during an acute attack of migraine. Particular emphasis has been directed towards the demonstration of catecholamines, monoamine oxidase, and cholinesterase activity in the vessels, because disturbed local synthesis or discharge of these agents might reasonably be considered as a possible precipitating factor in migraine.

\section{METHODS}

Biopsies of dilated superficial temporal arteries were obtained under local anaesthesia from six volunteer subjects during a severe attack of migraine and within a few hours from the onset of the attack. They had all suffered from recurrent severe migraine over many years (Table I) and had complained of localized pain and tenderness over a segment of the superficial temporal artery during attacks. These patients usually obtained relief with $2 \mathrm{mg}$ ergotamine tartrate taken either orally or by rectal suppository soon after the onset of the attack but, on this occasion, they had delayed such treatment until after the biopsy. Control biopsies were obtained at craniotomy from six non-migrainous subjects undergoing neurosurgical procedures. These patients were aged from 38 to 59 yr. All subjects were normotensive.

\section{TABLE I}

CLINICAL DETAILS OF PATIENTS WITH MIGRAINE

\begin{tabular}{|c|c|c|c|c|}
\hline & $\operatorname{Sex}$ & Age & $\begin{array}{l}\text { Length of History } \\
\text { of migraine } \\
(y r)\end{array}$ & Usual Therapy \\
\hline E.T. & $\mathbf{F}$ & 33 & 10 & Cafergot suppository \\
\hline B.P. & $\mathbf{F}$ & 40 & 15 & $\begin{array}{l}\text { Cafergot suppository, } \\
\text { Librium }\end{array}$ \\
\hline H.R. & $\mathbf{M}$ & 56 & 40 & $\begin{array}{l}\text { Cafergot suppository, } \\
\text { Migril }\end{array}$ \\
\hline B.R. & $\mathbf{F}$ & 43 & 11 & $\begin{array}{l}\text { Cafergot suppository, } \\
\text { Lingraine }\end{array}$ \\
\hline $\begin{array}{l}\text { R.S. } \\
\text { J.B. }\end{array}$ & $\begin{array}{l}\mathbf{M} \\
\mathbf{F}\end{array}$ & $\begin{array}{l}58 \\
55\end{array}$ & $\begin{array}{l}55 \\
28\end{array}$ & $\begin{array}{l}\text { Cafergot suppository } \\
\text { Migril, Drinamyl }\end{array}$ \\
\hline
\end{tabular}

The biopsied arteries were transported to the laboratory over ice in a Thermos flask. Part of each artery was fixed in $4 \%$ formaldehyde-saline at $4^{\circ} \mathrm{C}$, while the other part was rapidly frozen onto a cryostat chuck by means $\frac{\overline{\frac{\omega}{3}}}{2}$ of a carbon dioxide jet. Conventional cryostat sections $\Phi$ were used for dehydrogenase, phosphatase, and monoamine oxidase methods. For catecholamine studies, ${ }^{\infty}$ cryostat sections were either dehydrated within the $\vec{O}$ cryostat (that is, freeze-dried) for $\mathbf{4 0} \mathrm{min}$ or desiccated over solid carbon dioxide under reduced pressure $\vec{\omega}$ (Hamberger and Norberg, 1964); the former method was? found to be the most satisfactory in practice. The sections were warmed with the operator's hand before removing? them from the cryostat; this manoeuvre prevented $\omega$ condensation of water over them on exposure to laborz tory atmosphere.

Cholinesterase activity was demonstrated in cryos sections of tissue that had been fixed in cold formali 5 for $18 \mathrm{hr}$. The rest of the formalin-fixed material was embedded in paraffin for conventional microscopy.

Lactic dehydrogenase, reduced nicotinamide adenize dinucleotide-tetrazolium reductase $\left(\mathrm{NADH}_{2}-\mathrm{TR}\right)$ agd adenosine triphosphatase (ATPase) activities were demonstrated by methods previously described $\overrightarrow{0}$ Adams, Davison, and Gregson (1963). Monoamine oxidase activity was displayed by Glenner, Burtner, and Brown's (1957) method, with tryamine as substrate and nitroblue tetrazolium as the electron acceptor. Cholinesterase activities were demonstrated by Karnovsky and Roots' (1964) technique, with acetylthiocholine and butyrylthiocholine às substrates. The use of specific $\frac{\Phi}{\Phi}$ inhibitors of true and pseudocholinesterases proved to be unnecessary (see Results).

Catecholamines were detected by the ultraviolet 3 fluorescence of their isoquinoline derivatives after exposure to paraformaldehyde vapour at about $70 \%$ ? relative humidity in a closed container at $80^{\circ} \mathrm{C}$ for $2 \mathrm{hr}$ (Falck, 1962; Falck, Hillarp, Thieme, and Torp, 1962). For ultraviolet fluorescence microscopy, Leitz BG12 and Blau BG38 filters were used for excitation and a Leitz 3 $\mathrm{K} 530 \mathrm{~m} \mu$ filter was inserted as an ultraviolet barrier above the objective lens. The specificity of paraformalde- $O$ hyde-induced fluorescence for catecholamines was tested $₹$ by reducing the resulting isoquinolines to their non-을 fluorescent tetra-hydro derivatives with $0 \cdot 1$ sodium borohydride in $95 \%$ ethanol (Corrodi, Hillarp, ando Jonsson, 1964). The catecholamine-binding capacity of adrenergic nerve fibres was assessed after incubating $N$ cryostat sections in aqueous noradrenaline $(1 \mathrm{mg} / \mathrm{ml}$.) for 45 min (Angelakos and King, 1965). Some sections 
were preincubated in aqueous desipramine (Pertofran; $3 \mathrm{mg} / \mathrm{ml}$.) for $15 \mathrm{~min}$, before incubating for $45 \mathrm{~min}$ in a mixture of noradrenaline $(1 \mathrm{mg} / \mathrm{ml}$.) and desipramine (3 $\mathrm{mg} / \mathrm{ml}$.) (Carlsson, Fuxe, Hamberger, and Lindqvist, 1966). In order to determine the effect of ergotamine tartrate on periarterial adrenergic nerve fibres, some sections were incubated for $45 \mathrm{~min}$ in either ergotamine tartrate $(0.05 \mathrm{mg} / \mathrm{ml}$.) or ergotamine tartrate-noradrenaline mixture $(0.05 \mathrm{mg} / 1 \mathrm{mg} / \mathrm{ml}$.). Further to test this last point, a rat was injected subcutaneously with $0.03 \mathrm{mg}$ of this drug and was then killed $1 \mathrm{hr}$ later. The aorta from this rat and from an untreated animal were sectioned and examined for catecholamine fluorescence.

Selected fresh or formalin-fixed paraffin-embedded tissues were stained with haematoxylin and eosin, Verhoeff's elastic, Van Gieson, and Glees and Marsland's silver stains.

\section{RESULTS}

MORPHOLOGY No abnormalities are apparent in paraffin sections of migrainous temporal arteries. No inflammatory changes, oedema, thickening or proliferation are seen in the tunica media, while the medial muscle fibres and elastic laminae appear normal. The tunica adventitia is essentially normal, but may be slightly thickened with collagen (see Torda and Wolff, 1945; Wolff, 1963). The tunica intima is often diffusely thickened by collagen, smooth-muscle and elastic fibres. However, such intimal thickening is within the normal range for temporal arteries in normotensive subjects of the same age as the migraine patients (Ainsworth, Gresham, and Balmforth, 1961; Missen, 1963).

ENZYMES Lactic dehydrogenase, $\mathrm{NADH}_{2}$-TR, and ATPase activities are not altered in migrainous arteries. Cholinesterase is equally active in illdefined, very scanty adventitial nerve fibres of both control and migrainous arteries. Monoamine oxidase activity in medial smooth muscle fibres is somewhat variable; ranking the intensity of reaction shows no clear difference between control and migrainous vessels (see Table II; Figs. 1 and 2).

TABLE II

HISTOCHEMICAL MONOAMINE OXIDASE ACTIVITY IN TEMPORAL ARTERIES RANKED ACCORDING TO INTENSITY OF REACTION

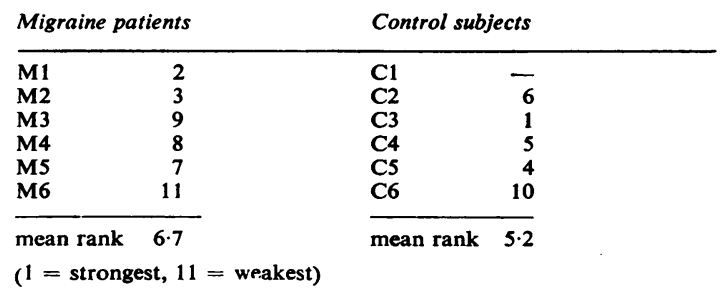

CATECHOLAMINES A number of fibres are revealed in the tunica adventitia with the paraformaldehyde fluorescence technique (Figs. 3 and 6). Such fibres disappear after immersing the section in either water or alcoholic borohydride (Fig. 4). After treatment with the latter reagent the fibres reappear when fluorescence is regenerated with paraformaldehyde. However, we were doubtful about the true nature of these fibres. Thus, they are refractile in ordinary transmitted light (Fig. 5) and frequently appear as parallel lines, neither of which features are characteristic of nerve terminals. These fibres could not be convincingly stained by either Glees and Marsland's axon stain or by Verhoeff's elastic method. Thus, the possibility arose that they may be the curled edges of dried collagen fibres. The apparent fluorescence of these refractile fibres is in fact artefactual; unlike the diffuse fluorescence of the main mass of adventitial collagen (see below), such artefactual fluorescence is extinguished when the section is mounted in liquid paraffin (Fig. 7). The extinction of artefactual 'fluorescence' engendered by immersion in water or alcoholic borohydride (Fig. 4) is perhaps due to flattening of collagen fibres after the section has been moistened. This explanation is strengthened by the observation that artefactual fluorescence reappears when the borohydride- or water-treated section is air-dried for some $30 \mathrm{~min}$. The reappearance of 'fluorescence' after further treatment with hot paraformaldehyde can thus be ascribed to the effects of drying.

COMMENT ON FLUORESCENCE ARTEFACTS It is clear that artefactual fluorescence is encountered when air-dried unmounted sections are viewed in ultraviolet light. If sections are to be subsequently treated with a blockading agent or stained for enzyme activity, it is obviously impractical to mount them in liquid paraffin or other non-fluorescent medium. Under these circumstances great care is needed to distinguish artefactual from real fluorescence. It should also be noted that arterial elastic laminae are fluorescent without exposure to paraformaldehyde, but such autofluorescence is unlikely to cause difficulty in interpretation.

CATECHOLAMINE-BINDING IN MIGRAINOUS ARTERIES Although the above-mentioned scattered 'fluorescent' fibres in the tunica adventitia are artefactual (Figs. 3 and 6), the main mass of adventitial collagen around migrainous arteries shows a marked capacity to absorb noradrenaline after incubating in this catecholamine. The diffuse fluorescence of the adventitia illustrated in Figs. 6-8 is characteristic of all six migrainous arteries, whereas the relative absence of such fluorescence-as shown in 


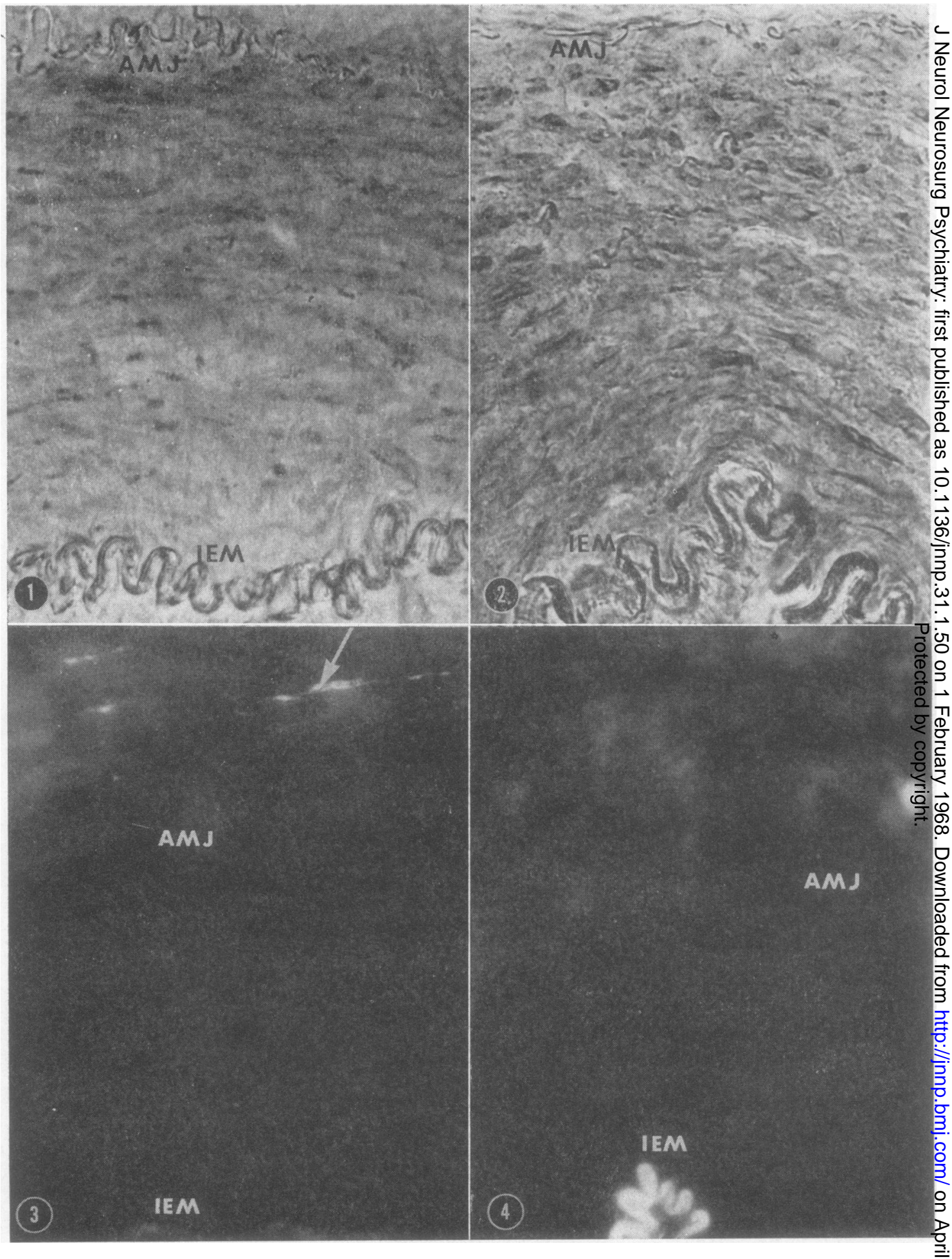

Symbols in the figures: IEM = internal elastic membrane, AMJ = adventitio-medial junction. In all illustrations the tunica adventitia is at the top and the tunica intima at the bottom.

FIG. 1. Monoamine oxidase activity in migrainous temporal artery. Tyramine-nitroblue tetrazolium, $\times 780$.

FIG. 2. As for Fig. 1 in control temporal artery.

FIG. 3. Trivial noradrenaline-binding in tunica adventitia of control temporal artery. Note artefactually fluorescent fibre (arrow). Incubated in noradrenaline, Falck's paraformaldehyde fluorescence method $\times 780$.

FIG. 4. Same artery as Fig. 3 but after inhibition of fluorescence by borohydride. Note that artefactual fibres have disappeared. 


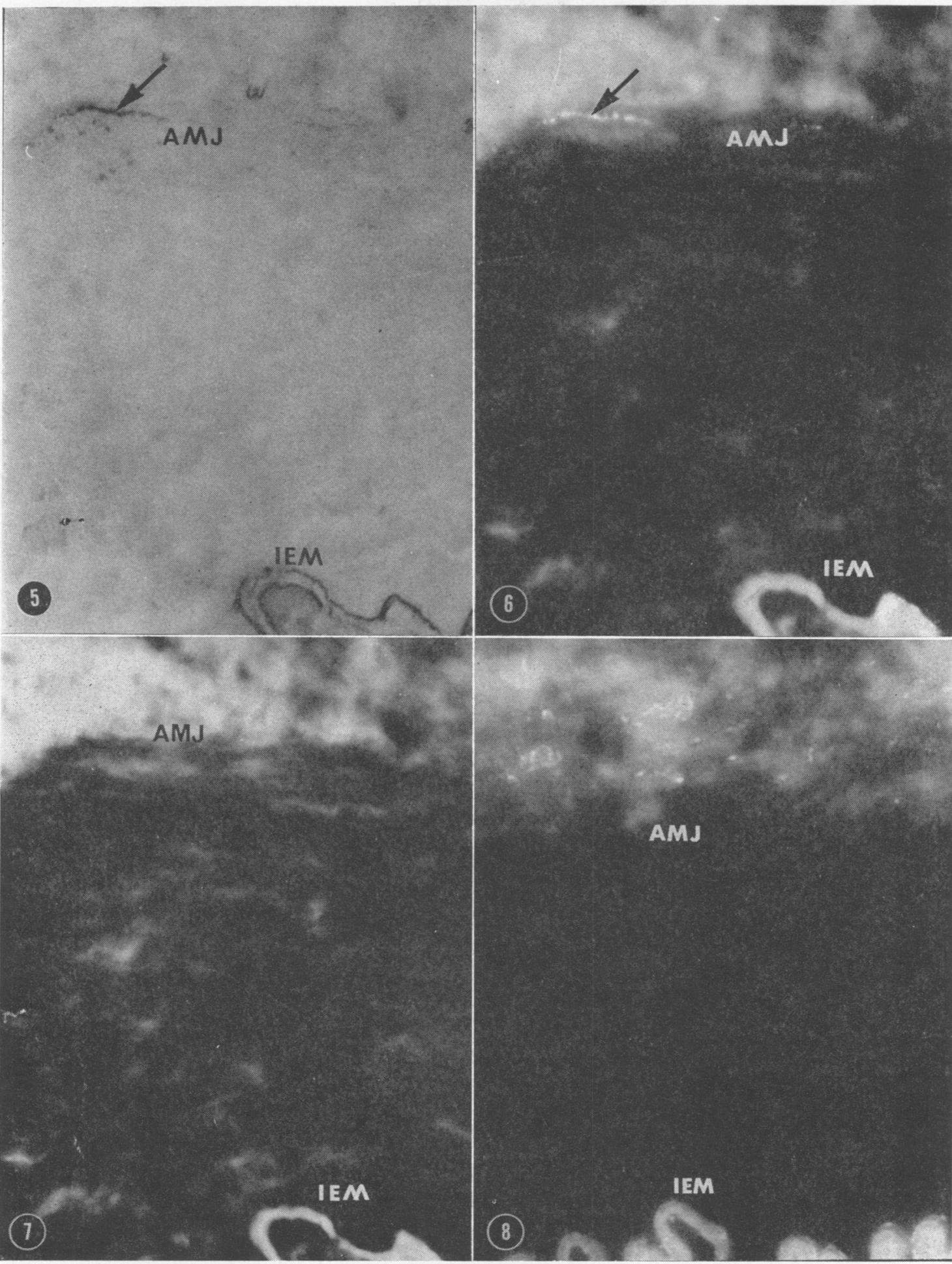

FIG. 5. Artefactual refractile fibre (arrow) at adventitio-medial junction. Same field as Fig. 6, but photographed in ordinary transmitted light $\times 780$.

FIG. 6. Strong noradrenaline-binding in tunica adventitia of migrainous temporal artery. Note artefactually fluorescent fibre (arrow) corresponding to that shown in Fig. 5. Incubated in noradrenaline, paraformaldehyde fluorescence method $\times 780$.

FIG. 7. Same field as Fig. 6, but mounted in liquid paraffin to show disappearance of artefactual fibre but persistence of diffuse fluorescence in tunica adventitia.

FIG. 8. Strong noradrenaline-binding in tunica adventitia of another migrainous temporal artery. Incubated in noradrenaline, paraformaldehyde fluorescence method $\times 780$. 
Fig. 3-is typical of all six control vessels. Such fluorescence is entirely inhibited by preliminary treatment with borohydride (Fig. 9) and can be partly regenerated by re-exposure to paraformalde-

hyde vapour (Fig. 10); it does not disappear when the section is mounted in liquid paraffin (Fig. 7). $Z$ It is irreversibly inhibited by immersion in water and $=$ does not reappear when the section is air-dried. \%

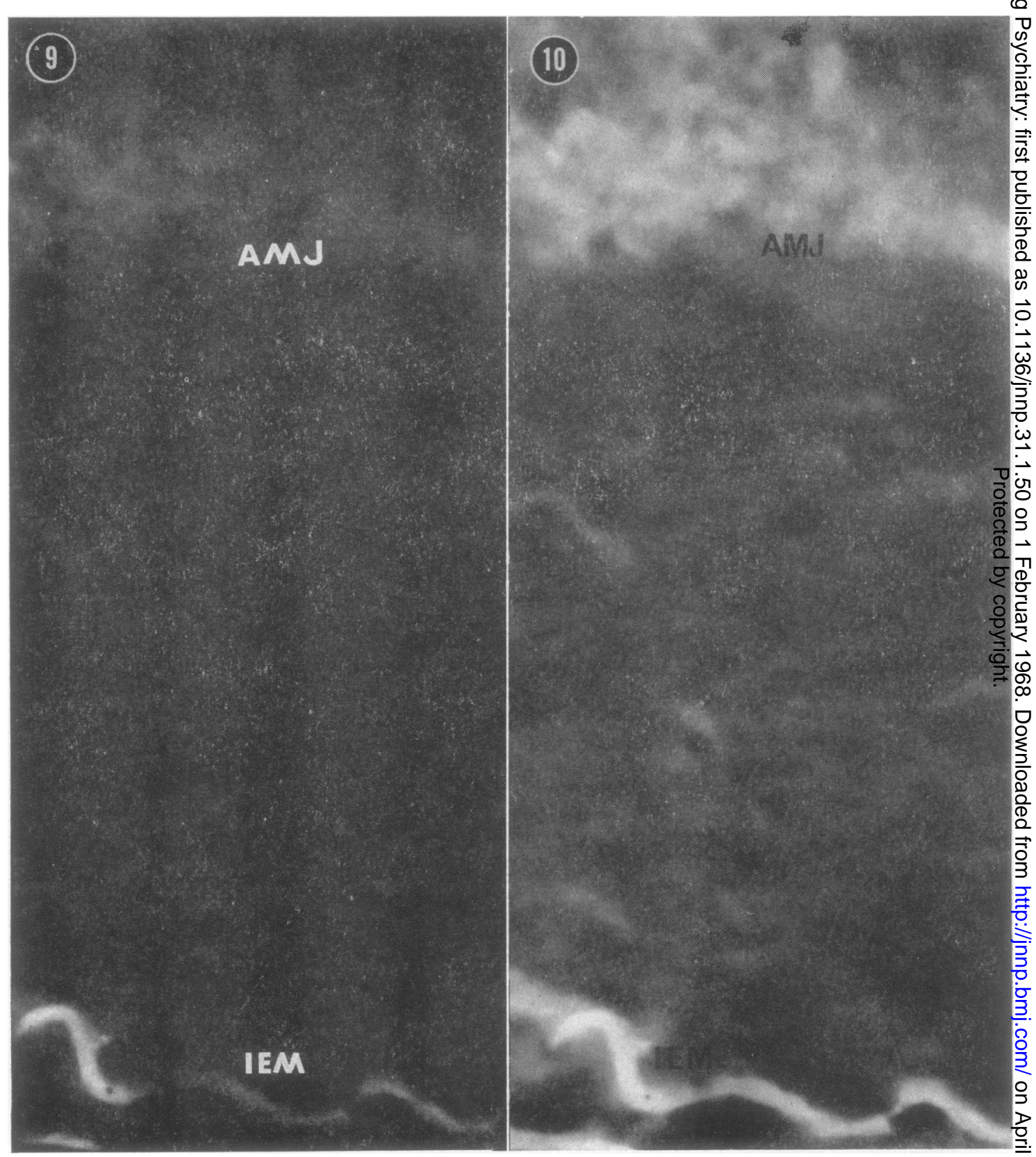

FIG. 9. Same tissue as Figs. 6 and 7 to show loss of paraformaldehyde induced fuorescence in tunica adventitia $N$ after treatment with borohydride $\times 960$.

FIG. 10. Same tissue as Fig. 9, but after regeneration of specific fluorescence by re-exposure to paraformaldehyde. 
Fluorescence is not altered by incubation of the artery in the desipramine-noradrenaline and ergotamine-noradrenaline mixtures. No paraformaldehydeinduced fluorescence is seen in the adventitia of either migrainous or control temporal arteries, when sections are not incubated in noradrenalinethat is, no endogenous catecholamines were detected with our technique in human temporal arteries. Incubation in ergotamine does not induce fluorescence.

In one additional case of migraine (and a corresponding control), we tested the effect of incubating sections in serotonin creatinine sulphate $(2 \mathrm{mg} / \mathrm{ml}$.): no convincing induced fluorescence is seen in either control or migrainous arteries.

CATECHOLAMINES IN RAT AORTA Fluorescent adrenergic fibres are seen in the adventitia and outer media of the rat aorta. Fluorescence is increased in sections incubated in noradrenaline, but some endogenous catecholamine is apparent in these fibres in unincubated tissue. Such fluorescence persists after the section is mounted in liquid paraffin, is extinguished by borohydride and is partially regenerated by re-exposure to paraformaldehyde. Injection of ergotamine into the rat does not alter the fluorescence in these aortic adventitial nerve fibres. However, the intensity of fluorescence is reduced in aortic sections incubated in a desipramine-noradrenaline mixture compared with those incubated in noradrenaline alone.

\section{DISCUSSION}

Histological examination of migrainous temporal arteries, excised during an acute attack, reveals no convincing structural abnormality in any of the vascular coats. Histoenzymic studies show that dehydrogenase, phosphoesterase, monoamine oxidase, and cholinesterase activities are unaffected. However, the paraformaldehyde-fluorescence method reveals that the tunica adventitia of migrainous arteries develops a striking increase in its binding capacity for noradrenaline after incubation of sections in this catecholamine.

It could be argued that this increased binding capacity is an adaptive response to repeated attacks of migraine involving the same segment of the temporalartery-such as had occurred in the patients from whom the biopsies were taken. This response could be considered as a local mechanism for storing noradrenaline to overcome the segmental dilatation of the migrainous temporal artery.

However, the increased adventitial capacity to bind noradrenaline in the migrainous artery is difficult to explain in terms of such a secondary response, for an increased level of noradrenaline in the arterial wall, such as is produced by injection of monoamine oxidase inhibitors, leads to the appearance of demonstrable extraneural catecholamine in the tunica media rather than the tunica adventitia (Doležel, 1966). Moreover, an adaptive catecholamine-storing response would be expected to be accompanied by hyperplasia of adrenergic nerve fibres, but no such neural proliferation was observed in the migrainous artery.

The balance of evidence suggests that, in the migrainous artery, noradrenaline released from nerve terminals at the adventitio-medial junction (see Adams, 1967) is unavailable to act on its targettissue - smooth-muscle in the tunica media-because it is bound by collagen in the tunica adventitia. Nevertheless, Gillespie (1966) reported that, while vascular smooth-muscle binds noradrenaline, normal elastin and collagen do not have this capacity.

Failure of noradrenaline to penetrate and act on the smooth-muscle of the tunica media would encourage dilatation of the arterial wall. Dilatation is characteristic of the temporal arteries in migraine, but the intracranial arteries are thought first to constrict and then dilate (Wolff, 1963). From the information available, the present very tentative hypothesis must, therefore, be restricted to events in the temporal arteries.

The anti-depressive agent desipramine (and related compounds) are known to impede in vitro noradrenaline uptake by adrenergic fibres around the rat aorta (see Results), by various nuclei in the rat brain and by adrenergic fibres in the rat vas deferens (Carlsson et al., 1966; see Glowinski and Axelrod, 1964). However, preincubation with this drug does not prevent in vitro uptake of noradrenaline by the tunica adventitia of the migrainous human temporal artery.

It may not be entirely fortuitous that migrainous headache responds to ergotamine. Moreover, the prophylactic use of a suitable ergotamine preparation can be very effective in avoiding migraine; the amine methysergide is also useful in this respect. However, ergotamine in our short-term in vitro studies did not affect the binding of noradrenaline by the arterial adventitia, so the success of the drug must be attributed to some other local or general mechanism.

In conclusion, it is clear that further studies are required in less florid and less chronic forms of migraine to determine whether or not the adventitial catecholamine-binding in migrainous temporal arteries is a primary or secondary event in this condition.

\section{SUMMARY}

The temporal arteries were biopsied in six patients while they were suffering from an acute attack 
of migraine and who complained of localized pain over a segment of the vessel. These biopsies reveal no significant structural change in the vessel wall. Histoenzymic methods do not disclose any significant change in dehydrogenase, phosphoesterase, monoamine oxidase, and cholinesterase activities in these arteries. However, the paraformaldehydefluorescence method shows that the tunica adventitia of migrainous arteries has a marked capacity to bind noradrenaline. This change may be a secondary response to repeated attacks of arterial dilatation in migraine. On the other hand, it may be a primary pathogenic event in migraine. The tentative hypothesis is presented that such adventitial catecholamine-binding may prevent noradrenaline from acting on the medial smooth-muscle fibres in the dilated migrainous temporal artery.

We are much indebted to the Abbeydale Trust and the British Migraine Association who partly supported this work. We also wish to express our thanks to Mr. J. J. Maccabe of King's College Hospital for providing the biopsies of normal temporal arteries, to the neurosurgical staff of the National Hospital, Queen Square, who performed the biopsies on migraine patients, to Mr. R.S. Morgan who prepared the photomicrographs, and Mr. J. F. C. Willder for histological assistance. Finally, we wish to acknowledge the cooperation of the six patients who volunteered to have their painful arteries biopsied during an attack of migraine.

\section{ADDENDUM}

It was pointed out to us during discussion at the Second Migraine Symposium (National Hospital for Nervous Diseases, London, 24 November 1967) that the migrainous arteries were obtained under local anaesthesia with procaine, whereas the control vessels were removed under general anaesthesia. In view of this comment we investigated the effect of incubating two necropsy specimens of temporal arteries in $2 \%$ procaine: this procedure did not increase the amount of subsequent noradrenaline-fluorescence in the tunica adventitia of $\subseteq$ these vessels.

\section{REFERENCES}

Adams, C. W. M. (1967). Vascular Histochemistry, ch. 2. Lloyd-Luke, $\stackrel{\vec{D}}{\rightarrow}$ London.

-, Davison, A. N., and Gregson, N. A. (1963). Enzyme inactivity of myelin: histochemical and biochemical evidence. J. Neuro- $\overline{\bar{C}}$
chem. 10, 383-395.

Ainsworth, R. W., Gresham, G. A., and Balmforth, G. V. (1961).ते Pathological changes in temporal arteries removed from $\varrho$ unselected cadavers. J. clin. Path., 14, 115-119.

Angelakos, E. T., and King, Mary. (1965). Histochemical demonstra- $=$ tion of catecholamine uptake by adrenergic nerve fibers. $\overrightarrow{0}$ J. Histochem. Cytochem., 13, 282-285.

Carlsson, A., Fuxe, K., Hamberger, B., and Lindqvist, M. (1966). Biochemical and histochemical studies on the effects of W imipramine-like drugs and (+)-amphetamine on central and? peripheral catecholamine neurons. Acta physiol. scand., 67, $481-497$.

Corrodi, H., Hillarp, N-A., and Jonsson, Gösta, (1964). Fluorescence? methods for the histochemical demonstration of monoamines. $\omega$ 3. Sodium borohydride reduction of the fluorescent compounds. as a specificity test. J. Histochem. Cytochem., 12, 582-586. T

Doležel, S. (1966). Histochemical identification of monoamine in theo arterial wall. Experientia (Basel), 22, 307.

Falck, B. (1962). Observations on the possibilities of the cellu@ir localization of monoamines by a fluorescence method. Aga physiol. scand., 56, suppl. 197.

-, Hillarp, N-Å., Thieme, G., and Torp, A (1962). Fluorescencęơf(D) catechol amines and related compounds condensed with formaldehyde. J. Histochem., Cytochem., 10, 348-354.

Gillespie, J. S. (1966). Tissue binding of noradrenalin. Proc. roy. B., 166, 1-10.

Glenner, G. G., Burtner, Helen J., and Brown, G. W. Jr. (1957). 审e histochemical demonstration of monoamine oxidase actipuy by tetrazolium salts. J. Histochem. Cytochem., 5, 591-600.

Glowinski, J., and Axelrod, J. (1964). Inhibition of uptake of tritiatednoradrenaline in the intact rat brain by imipramine ando structurally related compounds. Nature (Lond.), 204, 1318-1391.

Hamberger, B., and Norberg, K-A. (1964). Histochemical demonstration of catecholamines in fresh frozen sections. J. Histo-O chem. Cytochem., 12, 48-49.

Karnovsky, M. J., and Roots, L. (1964). A 'direct-coloring' thiocholine method for cholinesterases. J. Histochem. Cytochem., 12, 219-221.

Missen, G. A. K. (1963). The pathology of giant cell ('temporal') arteritis. D. M. Thesis, Oxford University.

Torda, C., and Wolff, H. G. (1945). Experimental studies on headache Arch. Neurol. Psychiat. (Chic.), 53, 329-332.

Wolff, H. G. (1963). Headache and Other Head Pain. 2nd ed., pp. 246307. Oxford University Press, New York. 